\title{
Video Article \\ Production and Measurement of Organic Particulate Matter in the Harvard Environmental Chamber
}

\author{
Yue Zhang ${ }^{1,2}$, Zhaoheng Gong ${ }^{1}$, Suzane de Sa ${ }^{1}$, Adam P. Bateman ${ }^{1}$, Yingjun Liu ${ }^{1}$, Yongjie Li ${ }^{1}$, Franz M. Geiger ${ }^{3}$, Scot T. Martin ${ }^{1,4}$ \\ ${ }^{1}$ School of Engineering and Applied Sciences, Harvard University \\ ${ }^{2}$ Department of Environmental Science and Engineering, Gillings School of Global Public Health, University of North Carolina \\ ${ }^{3}$ Department of Chemistry, Northwestern University \\ ${ }^{4}$ Department of Earth and Planetary Sciences, Harvard University
}

Correspondence to: Yue Zhang at yzhang01@live.unc.edu, Franz M. Geiger at geigerf@chem.northwestern.ed, Scot T. Martin at scot_martin@harvard.ed

URL: https://www.jove.com/video/55685

DOI: doi:10.3791/55685

Keywords: Environmental Sciences, Issue 141, Environmental Chamber, Particulate Matter (PM), Secondary Organic Material (SOM), Secondary Organic Aerosol (SOA), Aerosol Mass Spectrometer (AMS), Proton Transfer Reaction Mass Spectrometer (PTR-MS)

Date Published: $11 / 18 / 2018$

Citation: Zhang, Y., Gong, Z., Sa, S.d., Bateman, A.P., Liu, Y., Li, Y., Geiger, F.M., Martin, S.T. Production and Measurement of Organic Particulate Matter in the Harvard Environmental Chamber. J. Vis. Exp. (141), e55685, doi:10.3791/55685 (2018).

\section{Abstract}

The production and the evolution of atmospheric organic particulate matter (PM) are insufficiently understood for accurate simulations of atmospheric chemistry and climate. The complex production mechanisms and reaction pathways make this a challenging research topic. To address these issues, an environmental chamber, providing enough residence time and close-to-ambient concentrations of precursors for secondary organic materials, is needed. The Harvard Environmental Chamber (HEC) was built to serve this need, simulating the production of gas and particle phase species from volatile organic compounds (VOCs). The HEC has a volume of $4.7 \mathrm{~m}^{3}$ and a mean residence time of $3.4 \mathrm{~h}$ under typical operating conditions. It is operated as a completely mixed flow reactor (CMFR), providing the possibility of indefinite steady-state operation across days for sample collection and data analysis. The operation procedures are described in detail in this article. Several types of instrumentation are used to characterize the produced gas and particles. A High-Resolution Time-of-Fight Aerosol Mass Spectrometer (HRToF-AMS) is used to characterize particles. A Proton-Transfer-Reaction Mass Spectrometer (PTR-MS) is used for gaseous analysis. Example results are presented to show the use of the environmental chamber in a wide variety of applications related to the physicochemical properties and reaction mechanisms of organic atmospheric particulate matter.

\section{Video Link}

The video component of this article can be found at https://www.jove.com/video/55685/

\section{Introduction}

Atmospheric organic particulate matter (PM) is produced from the oxidation of volatile organic compounds (VOCs) emitted by the biosphere and anthropogenic activities ${ }^{1,2}$. Despite the important effects of these aerosol particles on climate, human health, and visibility ${ }^{3}$, the production mechanisms remain incompletely understood and characterized, both qualitatively and quantitatively. One challenge for laboratory studies, which are necessarily of limited scope and time, is to simulate the atmospheric evolution of gas and particle phase species. Residence times must be long enough that compounds in both gas and particles phases can undergo oxidation and multiphase reaction as they would in ambient environments ${ }^{4,5,6,7,8}$. Another challenge is to work in the laboratory at concentrations sufficiently low that represent the ambient environment ${ }^{9,10,11}$. Many important processes scale with concentrations. For instance, excessively high mass concentration of organic PM in a laboratory experiment can erroneously shift the partitioning of semivolatile species from the gas phase to the particle phase. The composition of the gas and particle phases can become non-representative of atmospheric conditions. The Harvard Environmental Chamber was designed to respond to these challenges, principally by using the approach of a continuous flow configuration operated under an indefinite timescale, thereby allowing low concentrations and long integration times for signal detection. The chamber celebrates a milestone anniversary of twelve years of scientific discovery in 2018.

Environmental chambers vary based on the light source, the flow mixing system, size, and the number of chambers operating together. There are outdoor chambers that receive natural sunlight ${ }^{12,13}$ as well as indoor chamber that operate with artificial light ${ }^{14,15,16,17,18,19,20,21}$. Outdoor chambers can also be built relatively large, minimizing artifacts that can be introduced by wall effects, although challenges include the variation of illumination because of clouds as well as variance in temperature. Although indoor chambers can carefully control temperature and relative humidity, the intensity and the spectrum from the artificial light are generally different from the natural sunlight, which may affect certain photochemical reactions ${ }^{14}$. Chambers can also be operated as batch reactors or completely mixed flow reactors (CMFR) ${ }^{22}$. Batch reactors are generally easier to operate and maintain but CMFR can be operated for weeks, as needed, to allow for signal integration and thereby work at low, atmospherically relevant concentrations. 
Herein, the hardware and the operation of the Harvard Environmental Chamber $(\mathrm{HEC})^{7,23,24,25}$ are described in detail. The HEC consists of a 4.7 $\mathrm{m}^{3}$ PFA Teflon bag housed inside a constant-temperature chamber $\left(2.5 \times 2.5 \times 2.75 \mathrm{~m}^{3}\right)^{26}$. Reflective aluminum sheets cover the inner walls of the chamber to allow multipath illumination through the bag and thereby increase the rate of photochemistry. The HEC is operated as a CMFR, using a total flow rate of $21 \mathrm{sLpm}$ and corresponding to a mean residence time of $3.4 \mathrm{~h}^{27}$. Temperature, humidity, and ozone concentration are maintained by feedback controls. Ammonium sulfate particles are used as seed particles to mimic the condensation of organic components onto inorganic particles in the ambient environment. The mode diameter of the inorganic sulfate particles is selected to be $100-200 \mathrm{~nm}$ to simulate the particle sizes measured in the field ${ }^{28}$. Operation procedures are described in the protocol section herein, including a visual presentation, followed by a brief discussion of applications and research results of the HEC.

\section{Protocol}

NOTE: The key measured environmental parameters include ozone (ozone analyzer), NO and NO2 (NOx analyzer), relative humidity (RH sensor), temperature (type $\mathrm{K}$ thermocouple), and the differential pressure between the bag and the chamber. The instrument brands are listed in the Table of Materials. The environmental parameters measured by the instruments must be stable and within design requirements prior to the start of the experiments. The environmental chamber uses a feedback system to constantly monitor and adjust controls so that the environmental parameters remain within requirements throughout the course of an experiment.

\section{Start Up Procedures}

1. Parameters Setting and oxidants injection

1. Set the physical parameters of the environmental chamber by the feedback system (PID). Set the differential pressure to $4 \mathrm{~Pa}$ ( 30 mTorr). When the pressure is too high or too low, the solenoid valve opens or closes to adjust the pressure of the bag within the set range.

2. Turn on the ozone generator to generate ozone flow by passing the dry air through an ultraviolet lamp. Set the flow rate to $0.1 \mathrm{sLpm}$ to reach $100 \mathrm{ppb}$ of final ozone concentration inside the HEC. Turn on the ozone monitor and activate the corresponding software.

3. Set the relative humidity of the bag to the designated values. In this particular experiment, use $40 \% \mathrm{RH}$ but the $\mathrm{RH}$ level can change from $<5 \%$ to $80 \%$. The $\mathrm{RH}$ sensor and the feedback control system keep the relative humidity in the bag stable by adjusting the ratio of dry and humid air flows. The dry air supply is provided by the pure air generator, which generates zero air free of hydrocarbons, water $(\mathrm{RH}<1 \%)$, and oxides of nitrogen. A flow of humid air is preparing by bubbling dry air through high purity water $(18 \mathrm{M} \Omega \mathrm{cm})$ to $\mathrm{create}$ near-saturated air flow.

4. Set the temperature of the chamber to $25.0 \pm 0.1^{\circ} \mathrm{C}$. An internal conditioning plenum distributes the air evenly through a stainless-steel ceiling with feedback control systems that keep the temperature within designated values.

5. Wait for other environmental parameters to be stable and within the design requirements.

2. Connect the inlets of instruments to the environmental chamber. Start the self-developed software by clicking the Start button. Check the real-time data displayed on the self-developed software that integrates the feedback control (Figure 2).

3. Turn on all the instruments and wait for them to warm up completely.

\section{Seed Particle Production}

NOTE: Prior to seed particle injection, the initial particle concentration is below $1 \mathrm{~cm}^{-3}$.

1. Production of sulfate seed particles

1. Inject quasi-monodisperse dry sulfate particles into the bag, both to better simulate the ambient conditions and also to act as medium for the condensational growth of secondary organic materials. The injection of seed particles is carried out as follows.

2. Dissolve ammonium sulfate $(0.01 \mathrm{~g})$ in high purity water $(18 \mathrm{M} \Omega \cdot \mathrm{cm})$ in a $100 \mathrm{~mL}$ volumetric flask to prepare a $(\mathrm{NH} 4) 2 \mathrm{SO} 4 \mathrm{solution}(0.1$ $\left.g \cdot L^{-1}\right)$.

3. Use an atomizer to produce (NH4)2SO4 aerosol particles at a flow rate of $3 \mathrm{sLpm}$.

4. Pass the aerosol flow through a diffusion dryer (silica gel) to bring the relative humidity down to $10 \%$.

2. Seed particle selection and bag injection

1. Pass the aerosol flow through a bipolar charger $\left({ }^{85} \mathrm{Kr}\right)$ and a Differential Mobility Analyzer (DMA) to size select the particles and prepare a quasi-monodisperse distribution by electric mobility. The transmission function is widened by using a sheath-to-aerosol flow in the DMA of 10:3. The selected electric mobility diameter of the particles exiting the DMA varies from 50 to $150 \mathrm{~nm}$ depending on the experiment. The typical number concentration ranges from 4,000 to $8,000 \mathrm{~cm}^{-3}$.

2. Feed the quasi-monodisperse aerosol to the bag with a flow rate of $3 \mathrm{sLpm}$. Monitor the particles exiting the bag by using a Scanning Mobility Particle Sizer (SMPS). Wait for the size distribution of the seed particles to become stable. The particle loss due to the bending tubing as well as static charges on the bag is minimal, especially for particles sizes used in this experiment (larger than $100 \mathrm{~nm})^{29,30}$.

\section{Injection of Gas Phase Precursors}

1. Injection of the gas-phase precursors

1. Use a syringe to withdraw $1.00 \mathrm{~mL}$ of the isoprene solution. Rinse the syringe three times with the solution prior to final withdrawal.

2. Place the syringe into a syringe injector. Insert the needle tip through a rubber seal into a round-bottom flask ( $25 \mathrm{~mL})$. Pre-heat the flask to $90 \pm 1^{\circ} \mathrm{C}$ by heating tape. Turn on the syringe injection and set it to an appropriate value $\left(1.1\right.$ to $\left.4.4 \mu \mathrm{L} \cdot \mathrm{min}^{-1}\right)$. The gas-phase concentration of the precursor is adjusted for different experiment by controlling the syringe injection rate. For long experiments, refresh the syringe as needed. 
3. Introduce a flow of $2 \mathrm{sLpm}$ of purified air to vaporize and carry away isoprene injected in the round-bottom flask. The flow of the air is large enough that the sessile droplet at the tip of the syringe is vaporized instead of dripping into the flask. As a result, the concentration of the gas phase precursor remains stable.

2. Turn on the switch of the ultraviolet lights inside the HEC.

\section{Instrument Measurement}

NOTE: The combination of isoprene and UV light leads to the production of secondary organic material onto the sulfate seed particles.

1. Number-diameter distribution of the particle exiting the bag

1. Sample the outflow from the bag by using the electrostatic-resistant tubing.

2. Start the aerosol measurement software and create a new file by clicking on Create A New File. Each parameter is set as shown in Figure 3. Record the number-diameter distributions of particles exiting the bag by clicking on the OK button.

2. Chemical characterization of organic particulate matter.

1. Open the sampling valve of a stainless steel inside the bag. The sampled aerosol flow in a High-Resolution Time-of-Flight Aerosol Mass Spectrometer (HR-ToF-AMS).

2. Start the data acquisition software by pressing the Acquire button on the lower left of the panel (Figure 4). High-resolution mass spectra of the organic PM are recorded during the time course of the experiments. The total organic mass concentration is also obtained.

3. Characterization of gas-phase species

1. Open the sampling valve of a PTFE Teflon tube inside the bag. The sampled flow is guided to a Proton-Transfer-Reaction Timeof-Flight Mass Spectrometer (PTR-TOF-MS). The mass spectra of gaseous species having a higher proton affinity than water are obtained.

2. Use the parameter settings of the ion source of the PTR-TOF-MS shown in Figure $\mathbf{5}$ in the software PTR-Manager. Start the data acquisition by accessing the drop-down menu Acquisition in the TofDAQ Viewer software and then pressing Start. Record the time series of each ion through this software.

\section{End of Experiment and Bag Cleaning}

1. Stop the injection of the gas-phase precursors and the aerosol seed particles.

2. For several days continuously inject pure air at $40 \mathrm{~L} \cdot \mathrm{min}-1$ into the bag. Turn on all ultraviolet lights. Set the ozone concentration to $600 \mathrm{ppb}$ and set the temperature to $40^{\circ} \mathrm{C}$. In this way, an aggressive oxidation environment is maintained for several days to scrub the bag. When the number concentration of the particles inside the chamber is less than $0.2 \mathrm{~cm}-3$, the chamber is considered clean and can be used for the next experiment.

\section{Representative Results}

An example of the time series of organic mass concentration recorded by the HR-ToF-AMS during one experiment is shown in Figure 6 .

Experimental conditions were $490 \mathrm{ppb}$ of isoprene with UV lights turning on to provide $\mathrm{OH}$ radical as oxidant. The mass concentration of organic PM steadily increased after the beginning of the experiment until after about $4 \mathrm{~h}$ steady state was reached. The evolution of gas-phase organic compounds was simultaneously studied using the PTR-TOF-MS. Figure 7 shows the time series of the $\mathrm{C}_{4} \mathrm{H}_{6} \mathrm{O}^{+}$signal intensity under the same experiment, which arises from major isoprene oxidation products (e.g., methyl vinyl ketone, methacrolein, and several organic hydroperoxides). After illumination was begun, the $\mathrm{C}_{4} \mathrm{H}_{6} \mathrm{O}^{+}$signal intensity increased and continued doing so until steady state was reached after 50 min.

The figures illustrate the time evolution of secondary organic material in the HEC. After injection, reaction, and spin up to steady state, the AMS data indicate that the particles are composed of organic compounds, and the concentration of the organic components increases with time. The PTR-MS data show that the parent precursor is lost from the gas phase and first-generation product species appear after the reaction is initiated. Data analysis of online and offline measurements typically focus on the steady-state period. There is the opportunity to conduct experiments that require days to finish because the concentrations of the gas and particle phase species remain stable indefinitely by use of the CMFR operation with feedback controls on important chamber parameters. In summary, the HEC is used to simulate atmospheric chemistry and thereby test hypotheses and understanding on important topics related to understanding air pollution, the climate influences of particles, and even health effects of aerosols. 


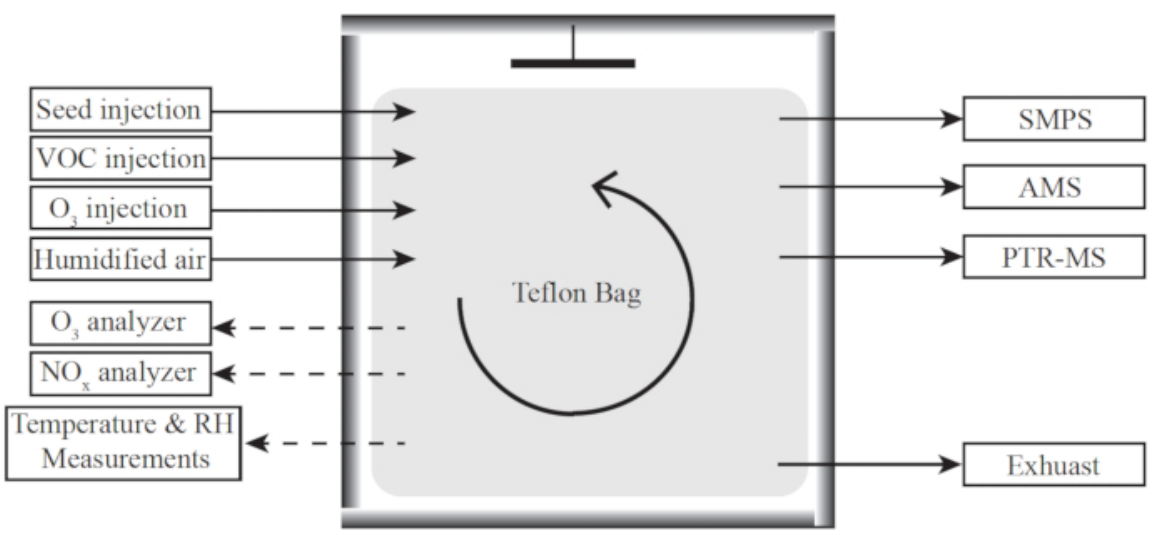

Figure 1. A schematic flow diagram of the Harvard Environmental Chamber (HEC). The lines represent the aerosol flow. The left panels show the instruments used to monitor reaction conditions. The right panels list those instruments used to characterize the particle and gas phase species. This figure is adapted from King et al. ${ }^{26}$ Please click here to view a larger version of this figure.

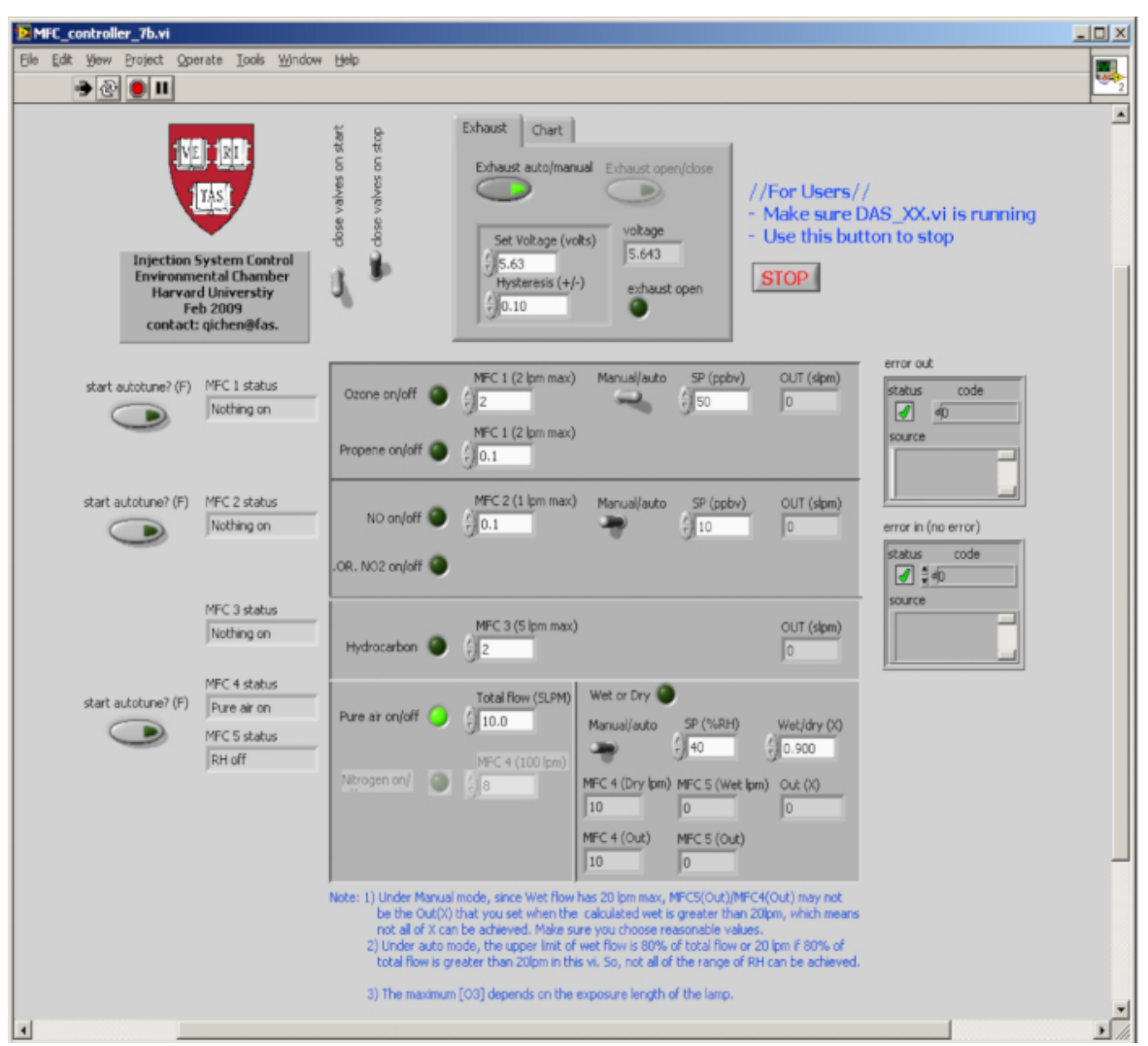

Figure 2. Graphical user interface for the self-developed program used to monitor temperature, ozone, relative humidity, and pressure, among other species. Please click here to view a larger version of this figure. 


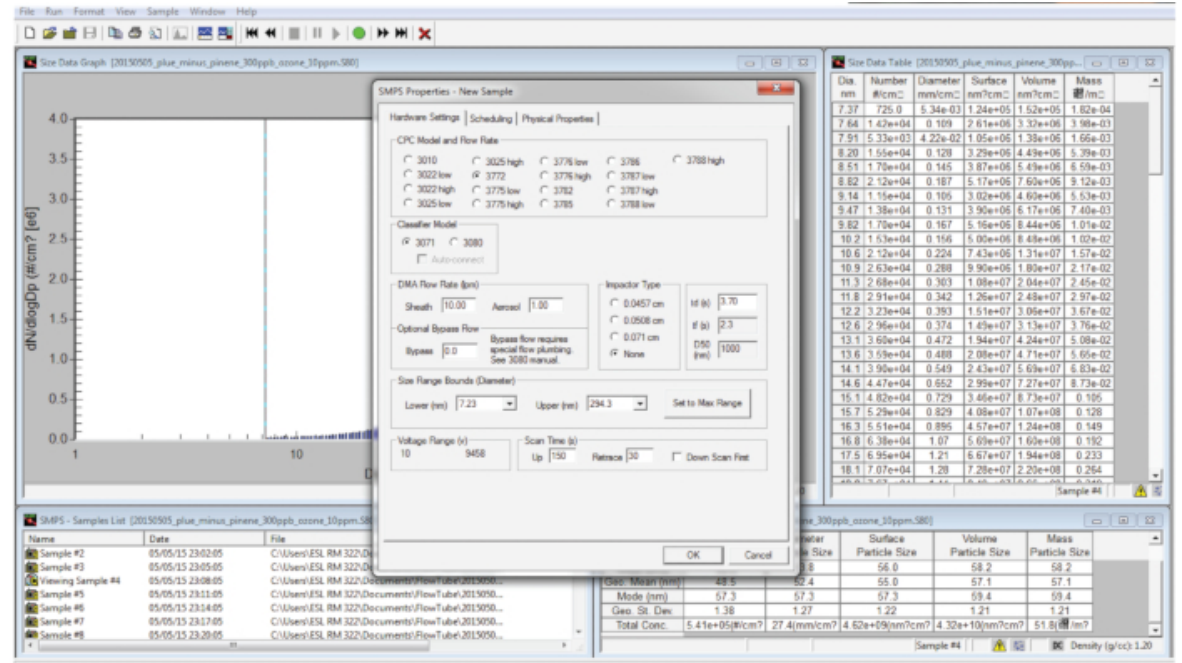

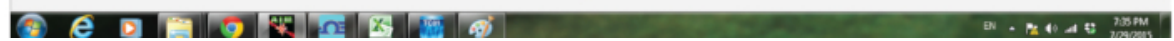

Figure 3. Graphical user interface of the software used to record the number-diameter distribution using a SMPS. Please click here to view a larger version of this figure.

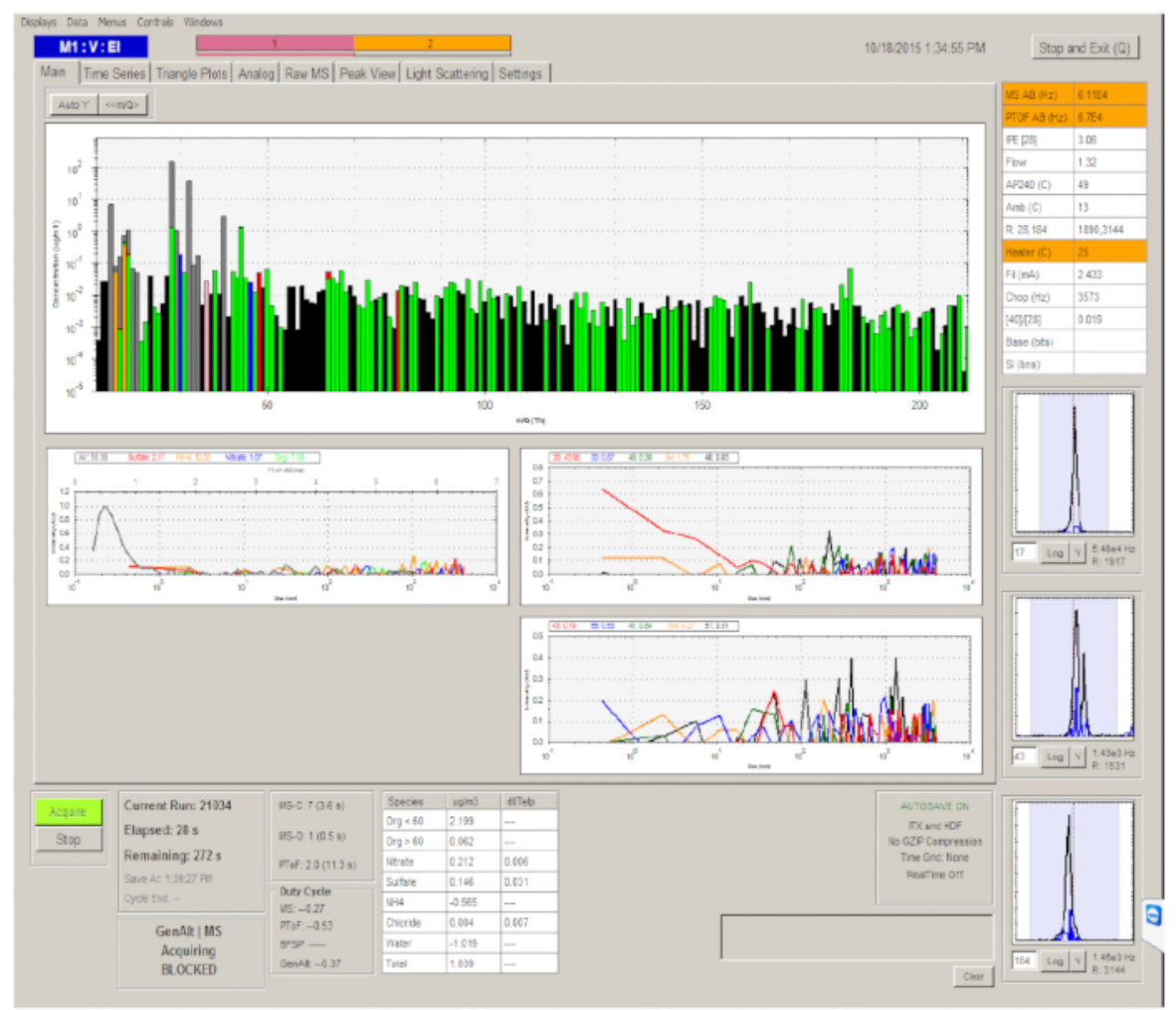

Figure 4. Graphical user interface of the software to operate the Aerosol Mass Spectrometer. Please click here to view a larger version of this figure. 


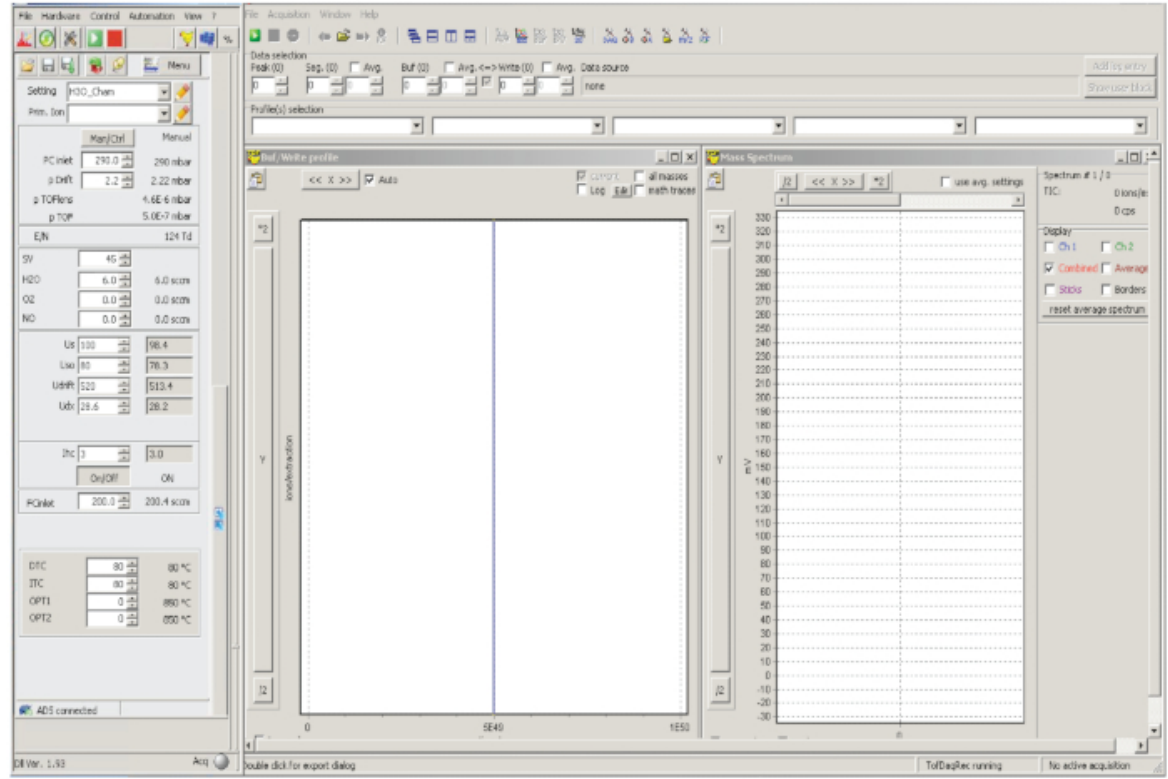

Figure 5. Graphical user interface of the software to control the Proton-Transfer-Reaction Mass Spectrometer (PTR-MS) Please click here to view a larger version of this figure.

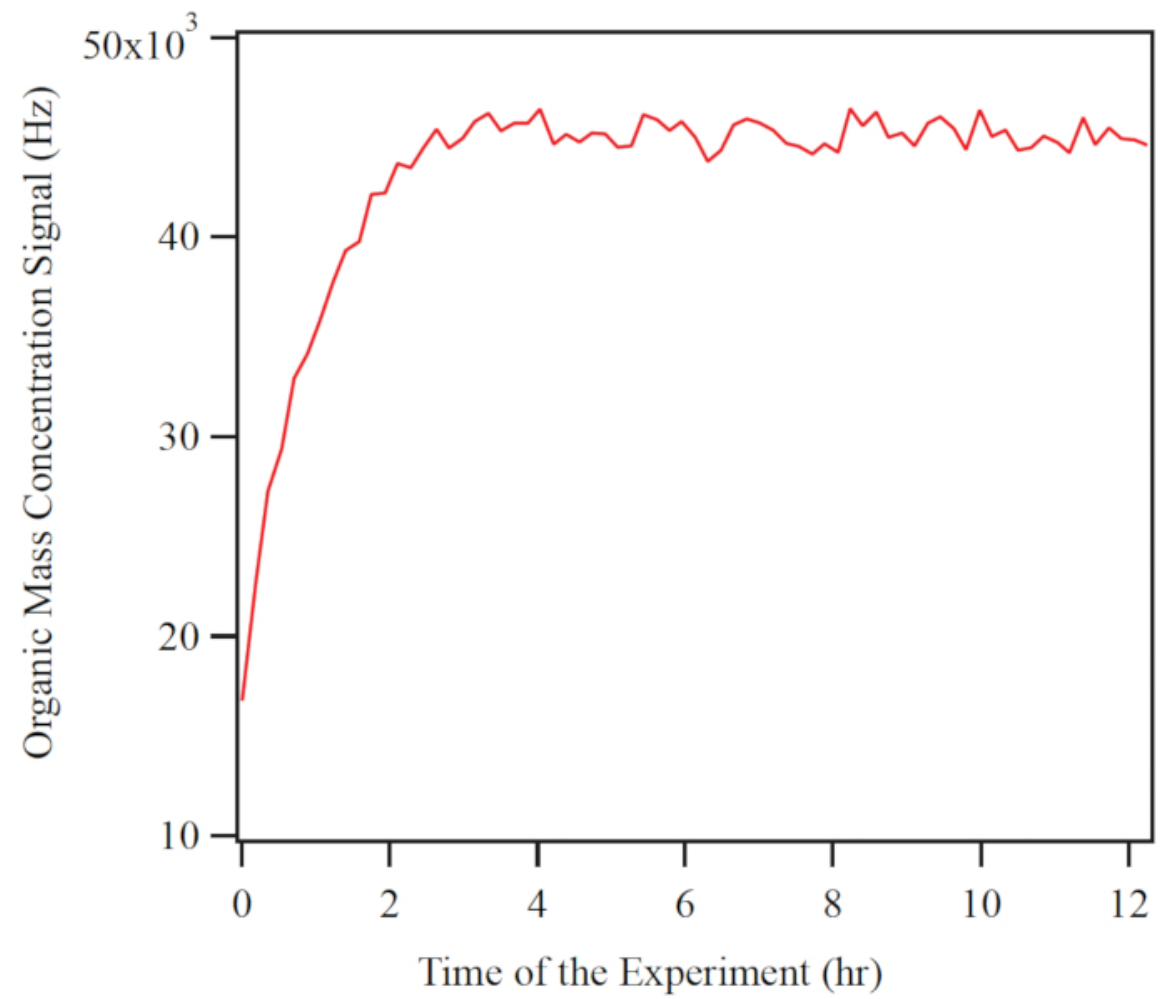

Figure 6. Example measurements for organic PM, as characterized by the Aerosol Mass Spectrometer. The red line represents the total signal intensity for organic ions. The shown data correspond to shortly after the isoprene was injected and the ultraviolet illumination commenced. Please click here to view a larger version of this figure. 


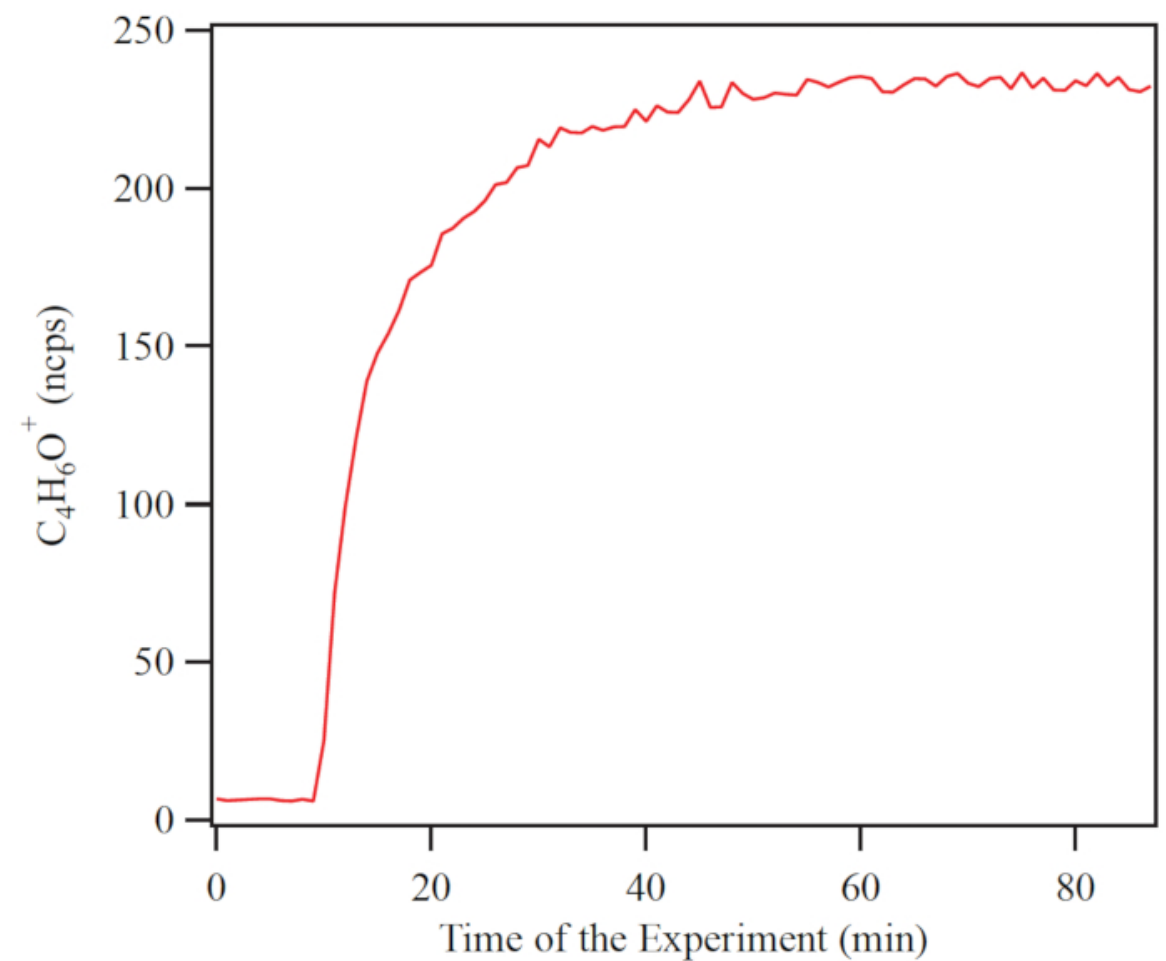

Figure 7. An example of the signal intensity of $\mathrm{C}_{4} \mathrm{H}_{6} \mathrm{O}^{+}$ion, a major product of isoprene photo-oxidation measurement is by ProtonTransfer-Reaction Mass Spectrometry. Signal intensity began to increase $8 \mathrm{~min}$ after illumination and reached steady state at 50 min. Please click here to view a larger version of this figure.

\section{Discussion}

The increasing significance in understanding the formation and evolution of organic aerosols leads to the urge to construct environmental chambers to simulate such processes in a well-controlled setting. At present, most of the environmental chambers are based on batch reactor mode ${ }^{19,31,32,33,34}$ while there have been very few chambers that utilize continuously mixing reactor mode ${ }^{15,35}$. Operating the environmental chamber in continuously flow reactor mode provides the convenience of continuous aerosol sampling for days or even weeks at ambient-like concentrations. It is worth noting that the ambient conditions are much more complex than the well-controlled laboratory settings. For instance, the temperature of ambient fluctuates while in the chamber it is maintained at a constant value. The reaction time of the gases and particles in the chamber will be controlled and limited by the residence of the chamber, rather than reaching days of reaction time in the real world. The use of blacklights, instead of natural solar radiation, can also generate $\mathrm{OH}$ radicals and simulate the reactions in the ambient. But blacklight sometimes can lead to elevated concentration of $\mathrm{OH}$ radicles compared with those in the ambient environment, which may affect the oxidation state of the organic molecules and needs to be carefully examined. However, by tuning only one or two variables and controlling all other variables through environmental chamber, we can systematically study these chemical/physical processes.

One of the critical steps in operating continuously mixing chambers is to keep the internal pressure of the chamber within an optimal range. A high pressure within the chamber will cause leaking of the gases and particles from the chamber, while a low pressure within the chamber will suck air and particles from the laboratory into the chamber and cause contamination. A pressure gauge is needed to monitor the pressure of the chamber within safe values $(<5 \mathrm{~Pa})$ throughout the course of the experiments. Another common observed issue for the environmental chamber is unexpected organic particle self-nucleation. Either a lower VOC/oxidant injection rate or a higher seed particle concentration is needed to avoid this phenomenon. Depending on the purpose of the experiments, the concentrations of ozone, VOC, and seed particles can vary by one order of magnitude. The following equation can be used to calculate the flow rate, $f_{\text {injection, }}$, of each species injected into the chamber.

$f_{\text {injection }}=\frac{c_{\text {target }}}{c_{\text {initial }}} f_{\text {total }}(1)$

where $c_{\text {target }}$ and $c_{\text {initial }}$ each represent the final target concentration of the reactant inside the chamber and the initial concentration of the reactant that is generated from the source. The symbol $f_{\text {total }}$ represent the total flow of all species that were injected into the chamber.

The third critical step for successfully operating the environmental chamber and obtaining the results is to calibrate each instrument before the experiments. The SMPS system can be calibrated by injecting known size of the PSL particles ${ }^{36}$. The $\mathrm{NO}_{\mathrm{x}}$ and ozone analyzer are calibrated by using a 5 ppm NO cylinder diluted by $\mathrm{N}_{2}$, and $10 \mathrm{ppm}$ of ozone diluted by $\mathrm{N}_{2}$, respectively ${ }^{26}$. The calibration procedures for AMS and PTR-MS are complicated and can be found in instrument manuals or previous literatures ${ }^{27,37}$. 
The environmental chamber setup described above is not only suitable for studying the production and evolution of organic aerosols, but also applicable in coating various particles with organic coating as well as examining gas phase reactions by injecting gas precursors only. These multiple directions provide environmental chamber the flexibility in studying a variety of research fields related to the air quality, climate change, and human health topics.

\section{Disclosures}

The authors declare no competing financial interests.

\section{Acknowledgements}

This material is based upon work supported by the Environmental Chemical Sciences Program in the Division of Chemistry of the U.S. National Science Foundation (NSF) under grant number 1111418, the Atmospheric-GeoSciences Division of the U.S. National Science Foundation (NSF) under grant number 1524731, as well as Harvard Faculty Publication Award. We acknowledge Pengfei Liu, Qi Chen, and Mikinori Kuwata for useful discussions and assistance with the experiments, as well as Eric Etcovitch for being the voiceover of the video.

\section{References}

1. Hallquist, M. et al. The formation, properties and impact of secondary organic aerosol: current and emerging issues. Atmospheric Chemistry and Physics. 9, 5155-5236 (2009).

2. Fehsenfeld, F. et al. Emissions of volatile organic compounds from vegetation and the implications for atmospheric chemistry. Global Biogeochemical Cycles. 6, 389-430 (1992).

3. Seinfeld, J. H., Pandis, S. N., Atmospheric Chemistry and Physics: from air pollution to climate change. John Wiley, Sons (2006).

4. Zaveri, R. A., Easter, R. C., Shilling, J. E., Seinfeld, J. H. Modeling kinetic partitioning of secondary organic aerosol and size distribution dynamics: representing effects of volatility, phase state, and particle-phase reaction. Atmospheric Chemistry and Physics. 14, 5153-5181 (2014).

5. Shiraiwa, M., Berkemeier, T., Schilling-Fahnestock, K. A., Seinfeld, J. H., Pöschl, U. Molecular corridors and kinetic regimes in the multiphase chemical evolution of secondary organic aerosol. Atmospheric Chemistry and Physics. 14, 8323-8341 (2014).

6. Ziemann, P. J., Atkinson, R. Kinetics, products, and mechanisms of secondary organic aerosol formation. Chemical Society Reviews. 41, 6582-6605 (2012)

7. Chen, Q., Liu, Y., Donahue, N. M., Shilling, J. E., Martin, S. T. Particle-Phase Chemistry of Secondary Organic Material: Modeled Compared to Measured O:C and H:C Elemental Ratios Provide Constraints. Environmental Science and Technology. 45, 4763-4770 (2011).

8. Liu, P., et al. (2018). Highly Viscous States Affect the Browning of Atmospheric Organic Particulate Matter. ACS Central Science. 4 (2) 207-215 (2018).

9. Jimenez, J. L. et al. Evolution of organic aerosols in the atmosphere. Science. 326, 1525-1529 (2009).

10. Goldstein, A. H., Galbally, I. E. Known and unexplored organic constituents in the earth's atmosphere. Environmental Science and Technology. 41, 1514-1521 (2007).

11. Martin, S. T. et al. Sources and properties of Amazonian aerosol particles. Reviews of Geophysics. 48, RG2002 (2010).

12. Zhang, H., Surratt, J. D., Lin, Y. H., Bapat, J., Kamens, R. M. Effect of relative humidity on SOA formation from isoprene/NO photooxidation: enhancement of 2-methylglyceric acid and its corresponding oligoesters under dry conditions. Atmospheric Chemistry and Physics. 11, 6411-6424 (2011).

13. Rohrer, F. et al. Characterisation of the photolytic HONO-source in the atmosphere simulation chamber SAPHIR. Atmospheric Chemistry and Physics. 5, 2189-2201 (2005).

14. Cocker, D. R., Flagan, R. C., Seinfeld, J. H. State-of-the-art chamber facility for studying atmospheric aerosol chemistry. Environmental Science and Technology. 35, 2594-2601 (2001).

15. Shilling, J. E. et al. Loading-dependent elemental composition of a-pinene SOA particles. Atmospheric Chemistry and Physics. 9, 771-782 (2009).

16. Presto, A. A., Huff Hartz, K. E., Donahue, N. M. Secondary organic aerosol production from terpene ozonolysis. 1. effect of UV radiation. Environmental Science and Technology. 39, 7036-7045 (2005).

17. Epstein, S. A., Blair, S. L., Nizkorodov, S. A. Direct photolysis of a-pinene ozonolysis secondary organic aerosol: effect on particle mass and peroxide content. Environmental Science and Technology. 48, 11251-11258 (2014).

18. Boyd, C. M. et al. Secondary organic aerosol formation from the $\beta$-pinene+NO3 system: effect of humidity and peroxy radical fate. Atmospheric Chemistry and Physics. 15, 7497-7522 (2015).

19. Xu, L., Kollman, M. S., Song, C., Shilling, J. E., Ng, N. L. Effects of NOx on the volatility of secondary organic aerosol from isoprene photooxidation. Environmental Science and Technology. 48, 2253-2262 (2014).

20. Loza, C. L. et al. Characterization of vapor wall loss in laboratory chambers. Environmental Science and Technology. 44, 5074-5078 (2010).

21. Lin, Y. H. et al. Isoprene epoxydiols as precursors to secondary organic aerosol formation: acid-catalyzed reactive uptake studies with authentic compounds. Environmental Science and Technology. 46, 250-258 (2012).

22. Martin, S. T., Kuwata, M., Smith, M. L. An analytic equation for the volume fraction of condensationally grown mixed particles and applications to secondary organic material produced in continuously mixed flow reactors. Aerosol Science and Technology. 48, 803-812 (2014).

23. Bateman, A. P., Bertram, A. K., Martin, S. T. Hygroscopic Influence on the Semisolid-to-Liquid Transition of Secondary Organic Materials. The Journal of Physical Chemistry A. 119, 4386-4395 (2015).

24. Shilling, J. E., King, S. M., Mochida, M., Worsnop, D. R., Martin, S. T. Mass spectral evidence that small changes in composition caused by oxidative aging processes alter aerosol CCN properties. Journal of Physical Chemistry A. 111, 3358-3368 (2007).

25. Smith, M. L., Kuwata, M., Martin, S. T. Secondary Organic Material Produced by the Dark Ozonolysis of alpha-Pinene Minimally Affects the Deliquescence and Efflorescence of Ammonium Sulfate. Aerosol Science and Technology. 45, 244-261 (2011). 
26. King, S. M., Rosenoern, T., Shilling, J. E., Chen, Q., Martin, S. T. Increased cloud activation potential of secondary organic aerosol for atmospheric mass loadings. Atmospheric Chemistry and Physics. 9, 2959-2971 (2009).

27. Shilling, J. E. et al. Particle mass yield in secondary organic aerosol formed by the dark ozonolysis of a-pinene. Atmospheric Chemistry and Physics. 8, 2073-2088 (2008).

28. Nguyen, T. K. V. et al. Trends in particle-phase liquid water during the Southern Oxidant and Aerosol Study. Atmospheric Chemistry and Physics. 14, 10911-10930 (2014).

29. Nah, T., McVay, R. C., Pierce, J. R., Seinfeld, J. H., Ng, N. L. Constraining uncertainties in particle-wall deposition correction during SOA formation in chamber experiments. Atmospheric Chemistry and Physics. 17, 2297-2310 (2017).

30. Reineking, A., Porstendörfer, J. Measurements of Particle Loss Functions in a Differential Mobility Analyzer (TSI, Model 3071 ) for Different Flow Rates. Aerosol Science and Technology. 5, 483-486 (1986).

31. Kroll, J. H., Seinfeld, J. H. Chemistry of secondary organic aerosol: Formation and evolution of low-volatility organics in the atmosphere. Atmospheric Environment. 42, 3593-3624 (2008).

32. Surratt, J. D. et al. Reactive intermediates revealed in secondary organic aerosol formation from isoprene. Proceedings of the National Academy of Sciences USA. 107, 6640-6645 (2010).

33. Surratt, J. D. et al. Effect of Acidity on Secondary Organic Aerosol Formation from Isoprene. Environmental Science and Technology. 41, 5363-5369 (2007)

34. Isaacman-VanWertz, G. et al. Chemical evolution of atmospheric organic carbon over multiple generations of oxidation. Nature Chemistry. 10, 462-468 (2018).

35. Docherty, K. S. et al. Trends in the oxidation and relative volatility of chamber-generated secondary organic aerosol. Aerosol Science and Technology. 1-13 (2018).

36. Wiedensohler, A. et al. Mobility particle size spectrometers: Calibration procedures and measurement uncertainties. Aerosol Science and Technology. 52, 146-164 (2018).

37. Warneke, C., de Gouw, J. A., Kuster, W. C., Goldan, P. D., Fall, R. Validation of Atmospheric VOC Measurements by Proton-TransferReaction Mass Spectrometry Using a Gas-Chromatographic Preseparation Method. Environmental Science and Technology. 37, 2494-2501 (2003). 